\title{
Comparative study of the therapeutic potential of octreotide versus trimetazidine and their combination on acute pancreatitis in male rats
}

\author{
Sameer H. Al-Rekabi, ${ }^{a}$ Hassan A. Al-Aqooli, ${ }^{a}$ and Muayed S. Salimª ${ }^{*}$
}

Department of Pharmacology and Therapeutics, College of Medicine, Kufa University, Iraq.

*Correspondence to Muayed S. Salim (email: muayedsalih66@gmail.com).

(Submitted: 16 August 2018 - Revised version received: 02 September 2018 - Accepted: 05 September 2018 - Published online: 26 December 2018)

\begin{abstract}
Objective Acute pancreatitis continues to be associated with significant rates of mortality and morbidity, and therapeutic options are still very limited. Various theories have been suggested regarding the pathophysiology of acute pancreatitis, lot of research into different medical treatments for the treatment of acute pancreatitis, but it is not clear what benefits each treatment has, or indeed if any medical treatment is beneficial apart from supportive treatment.

Aim To clarify the potential therapeutic effect of octreotide, trimetazidine, and their combination in acute pancreatitis.

Methods Acute pancreatitis was induced by L-arginine and treated with octreotide subcutaneously, trimetazidine intraperitoneally and combination therapy by octreotide and trimetazidine. The rats were followed for $24 \mathrm{~h}$. At the $24^{\text {th }}$ hour we determined serum levels of tumor necrosis factor- $a$ (TNF- $a$ ), interleukin-1 $\beta$ (IL-1 $\beta$ ), total antioxidant capacity (TAC), lipase, and amylase, and the pancreatic tissues were analyzed histopathologically.

Results TNF- $a(P<0.001)$, IL-1 $\beta(P<0.001)$, TAC $(P<0.001)$, lipase $(P<0.001)$, and amylase $(P<0.001)$ serum levels and scores of histopathological changes $(P<0.05)$ were significantly lower in combination group as compared with both octreotide and trimetazidine groups.

Conclusion Combination treatment markedly decreases biochemical and histopathological changes in acute pancreatitis, thus ameliorate pancreatic injury in L-arginine induced acute pancreatitis.

Keywords acute, pancreatitis, arginine, octreotide, trimetazidine
\end{abstract}

\section{Introduction}

Acute pancreatitis (AP) is defined as an acute inflammatory process of the pancreas that usually associated with a severe pain in the upper abdomen; in most instances, blood levels of pancreatic enzymes, including amylase and lipase, are increased to at least three times the upper limit of normal. The inflammatory reaction in AP results in edema of the pancreas and extensive local and systemic effects. AP may occur as a new event or as a recurrent condition. In about $85 \%$ of cases, the disease takes a mild course with complete disappearance of clinical symptoms in a few days. In a small group of about $15-20 \%$ of patients, the disease takes a severe course, necrotising pancreatitis. This may culminate in multi-organ failure and death in about $15-40 \%$ of these patients. ${ }^{1}$

Acute pancreatitis continues to be associated with significant rates of mortality and morbidity, and therapeutic options are still very limited. Various theories have been suggested regarding the pathophysiology of acute pancreatitis, lot of research into different medical treatments for the treatment of acute pancreatitis, but it is not clear what benefits each treatment has, or indeed if any medical treatment is beneficial apart from supportive treatment. ${ }^{2}$

\section{Materials and Methods}

\section{Experimental Animals}

A total of 45 Sprague Dawley adult male rats weighing 200-300 g, were purchased from Animal Resource Center, Veterinary Medicine College, Al-Qassim University, the National Center for Drug Control and Researches, Iraq. The animals looked healthy and they lived in the animal house of College of Pharmacy, University of Kufa in a temperature ranged $\left(25 \pm 2^{\circ} \mathrm{C}\right)$ at room temperature, with vacillating 12 -h light/12-h dark cycles and the animals were permitted for free access to water and chow diet until the beginning of the study.

\section{Study Design}

Rats were randomized into five groups $(n=9)$ in each group as follows: Control group: injected with normal saline with two doses of $1.25 \mathrm{ml} / 100 \mathrm{~g}$ separated by 1 -h interval, and injected with normal saline in an equivalent volume to trimetazidine and octreotide solutions. Induction group: injected with L-arginine with two doses of $250 \mathrm{mg} / 100 \mathrm{~g}$ separated by 1 -h interval, and injected with normal saline in an equivalent volume to trimetazidine and octreotide. Octreotide treated group: injected with single octreotide dose of $4 \mu \mathrm{g} / \mathrm{kg}$ s.c. after induction with L-arginine. Trimetazidine treated group: injected with single trimetazidine dose of $10 \mathrm{mg} / \mathrm{kg}$ i.p. after induction with L-arginine. And combination group: injected with single trimetazidine dose of $10 \mathrm{mg} / \mathrm{kg}$ i.p. and single octreotide dose of $4 \mu \mathrm{g} / \mathrm{kg}$ s.c. after induction with L-arginine.

\section{Induction of Acute Pancreatitis}

After weighing the animal accurately, we calculated the volume of L-arginine hydrochloride solution (20\%) to be injected using a dose of $250 \mathrm{mg} / 100 \mathrm{~g}$ body weight $(1.25 \mathrm{ml} / 100 \mathrm{~g})$.

Then filling the solution in a sterile of $2 \mathrm{ml}$ syringe with 25 G 5/8" needle and inject i.p. and the animal was put in a clean cage with food and water. We do the injection in a laminar 
air flow hood with filters. After waiting for $1 \mathrm{~h}$, the $2^{\text {nd }}$ dose of i.p. was then delivered and the animal was again returned to its cage. Rats become slow after the $2^{\text {nd }}$ injection but gradually recovers.

\section{ELIZA}

Following the induction of acute pancreatitis after $24 \mathrm{~h}$, the blood was drawn using direct needle puncture of the heart. Samples of the blood were left to clot at $37^{\circ} \mathrm{C}$ and centrifuged at $3000 \mathrm{rpm}$ for $10 \mathrm{~min}$; serum was pulled, and analyzed for the detection of serum lipase, amylase, interleukin- $1 \beta$ (IL-1 $\beta$ ), tumor necrosis factor- $\alpha$ (TNF- $\alpha$ ) and total antioxidant capacity (TAC). The pancreas of the rat was removed and fixed for histopathological analysis.

\section{Histological Examination}

The entire pancreas was immediately removed and fixed in $10 \%$ formalin, processed by routine histological methods and embedded in paraffin blocks. About $5 \mu \mathrm{m}$ thick sections were cut and stained with hematoxylin-eosin (H\&E) for subsequent histological examination. After fixation, evaluation of scores were carried out by a histopathologist who was evaluated blindly to the experimental treatment groups. The degree of pancreatic damage and photographs were obtained from each pancreatic section ( $n=3$ sections per sample) under optical microscope. The morphological criteria that was used to assess the histopathological status were: Edema, acinar necrosis, hemorrhage and fat necrosis, and Inflammation, according to a scoring system used by Schmidt et al. ${ }^{4}$ and Yenicerioglu et al.

\section{Results}

At the end of this study, it is found that serum TNF- $\alpha$, IL- $1 \beta$, interleukin-6 (IL-6), TAC, lipase, and amylase serum levels and scores of histopathological changes were significantly elevated in induction group as compared with that of control group. Both octreotide and trimetazidine significantly counteract the increase in serum levels of TNF- $\alpha$, IL- $1 \beta$, TAC, lipase, and amylase. Histological analysis revealed that both octreotide or trimetazidine markedly reduced the severity of pancreatic injury in rats underwent $\mathrm{L}$-arginine induction procedure.

While combination therapy of octreotide plus trimetazidine decrease these parameters significantly as compared with induction group and even with octreotide or trimetazidine groups.

Results are shown in Figs. 1-10.

\section{Discussion}

Acute pancreatitis is an inflammatory condition of the pancreas, with varying involvement of other tissues and organs. The disease includes a spectrum of pancreatic lesions, varying from parenchymatous edema to severe hemorrhagic pancreatitis, with necrosis, infection and organ destruction. ${ }^{6}$

Acute pancreatitis is a disease with high morbidity and mortality. Various theories have been suggested regarding the pathophysiology of acute pancreatitis, yet the underlying mechanism is not clearly understood. Oxygen free radicals (OFRs) and basic proinflammatory cytokines, such as TNF- $\alpha$,
IL- $1 \beta$ and IL- 6 , which play a role in acute pancreatitis and other systemic inflammatory conditions, have been suggested to be responsible for the local tissue damage and multiple organ failure that occur during acute pancreatitis. The OFRs lead to membrane lipid peroxidation, changes in the main components of the cytoplasm and early activation of digestive enzymes of pancreatitis, and they initiate protein damage in acute pancreatitis.

\section{Effect of Acute Pancreatitis on Study Parameters after I-Arg Induction}

The present study shows significant elevation $(P<0.001)$ in serum level of proinflammatory cytokines (TNF- $\alpha$ and IL-1 $\beta$ ) in induction group as compared with the control group. Similar results obtained by Wang et al. ${ }^{7}$ mentioning that in AP, TNF- $\alpha$ and IL- $1 \beta$ may play an important role in stimulating

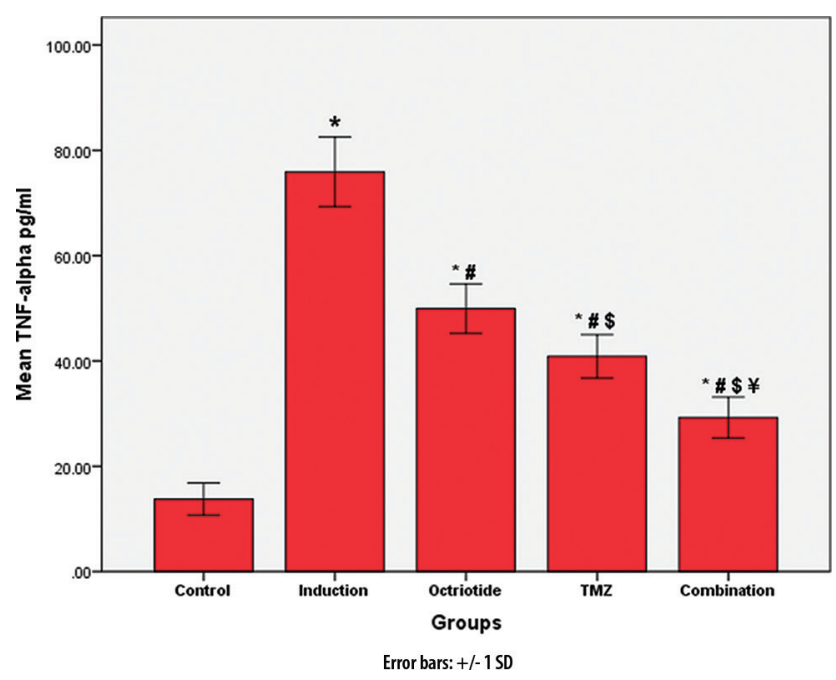

Fig. 1 The mean level of TNF- $\alpha(\mathrm{pg} / \mathrm{ml})$ in the five experimental groups. "Significant vs control group, "significant vs induction group, ${ }^{,}$significant vs octreotide, ${ }^{*}$ significant vs TMZ. Data are expressed as mean \pm SD.

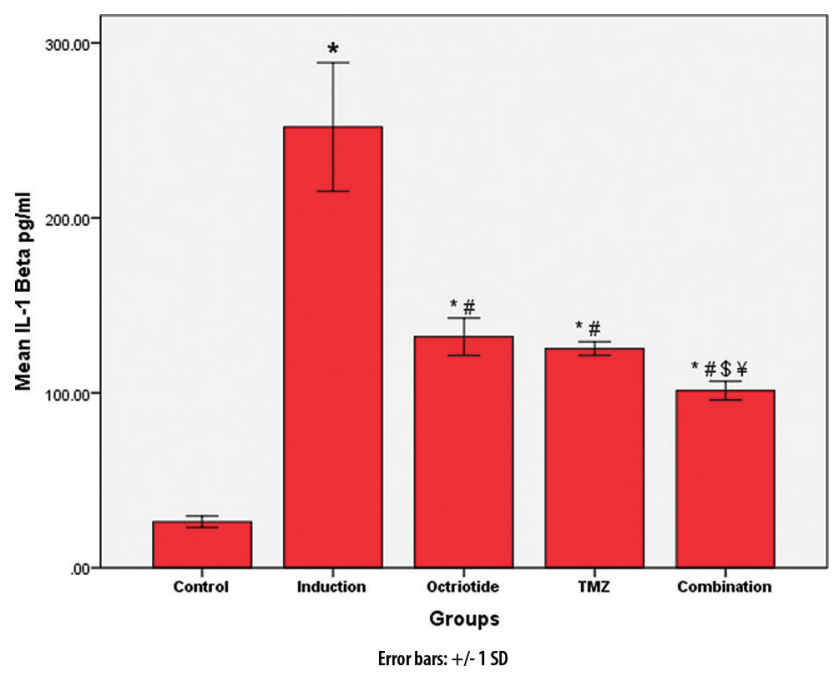

Fig. 2 The mean level of IL- $1 \beta(\mathrm{pg} / \mathrm{ml})$ in the five experimental groups. "Significant vs control group, ${ }^{\#}$ significant vs induction group, "significant vs octreotide, ${ }^{*}$ significant vs TMZ. Data are expressed as mean \pm SD. 


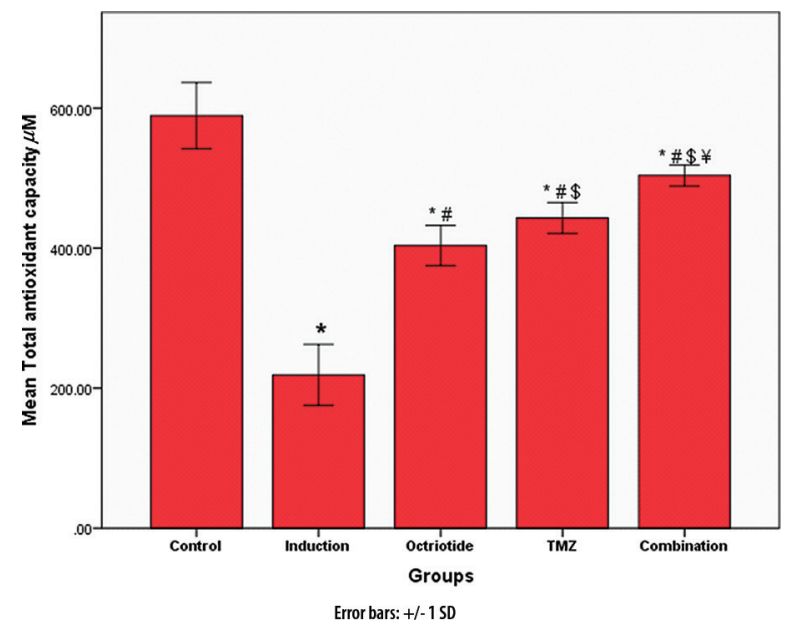

Fig. 3 The mean level of $\operatorname{TAC}(\mu \mathrm{M})$ in the five experimental groups. "Significant vs control group, "significant vs induction group, ssignificant vs octreotide, ${ }^{*}$ significant vs TMZ. Data are expressed as mean $\pm S D$.

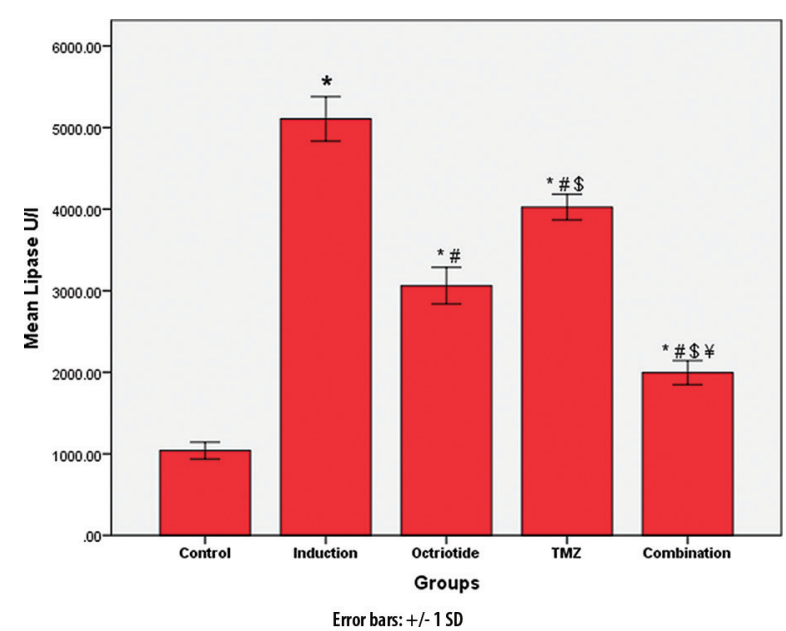

Fig. 4 The mean level of lipase (U/L) in the five experimental groups. "Significant vs control group, "significant vs induction group, ${ }^{\dagger}$ significant vs octreotide, ${ }^{*}$ significant vs TMZ. Data are expressed as mean \pm SD.

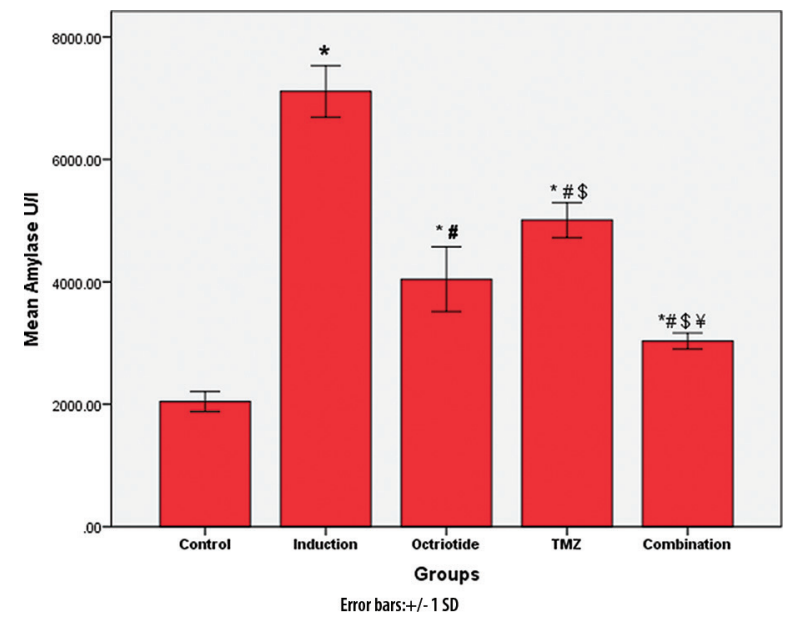

Fig. 5 The mean level of amylase $(\mathrm{U} / \mathrm{L})$ in the five experimental groups. "Significant vs control group, "significant vs induction group, ssignificant vs octreotide, ${ }^{\sharp}$ significant vs TMZ. Data are expressed as mean $\pm S D$.

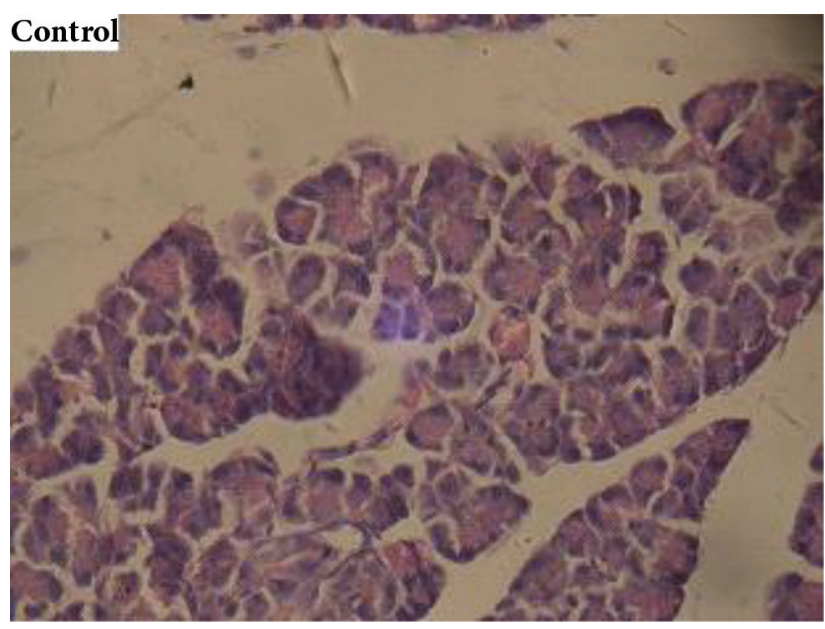

Fig. 6 Histopathological changes in pancreatic tissue regarding edema, acinar necrosis, hemorrhage and fat necrosis, and inflammation.

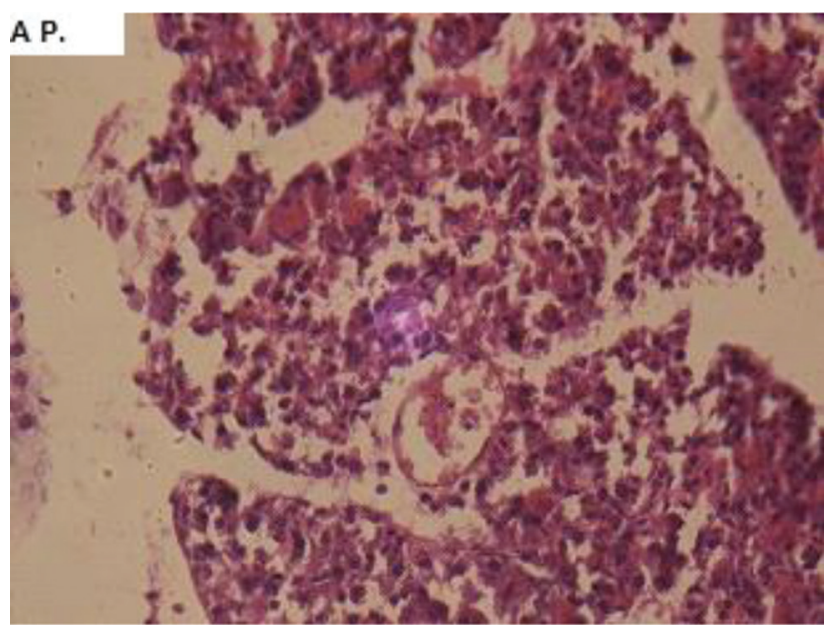

Fig. 7 Edema scoring in different experimental groups.

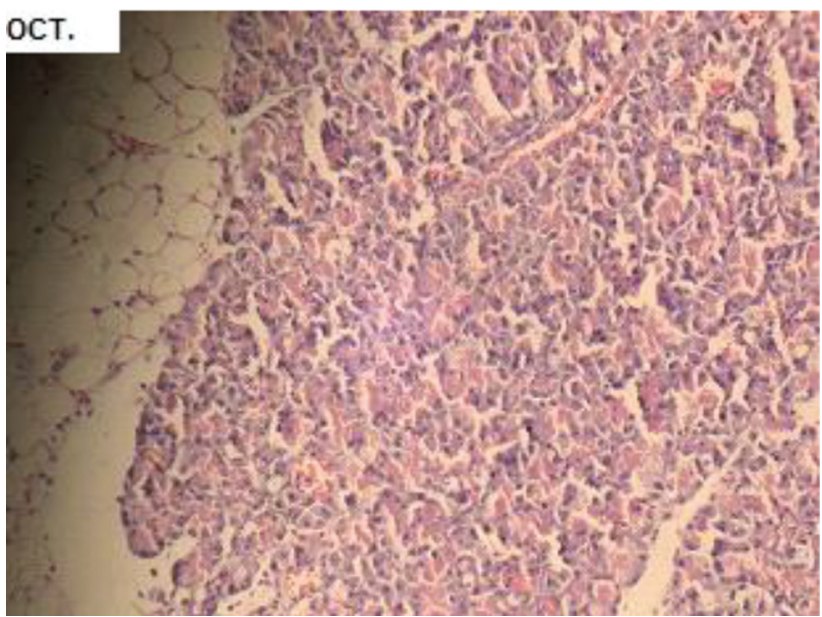

Fig. 8 Acinar necrosis scoring in different experimental groups.

the inflammatory response in the animal experimental studies, the levels of IL- $1 \beta$ and TNF- $\alpha$ increased significantly in the blood and in pancreatic tissues, and associated with the severity of the disease. 


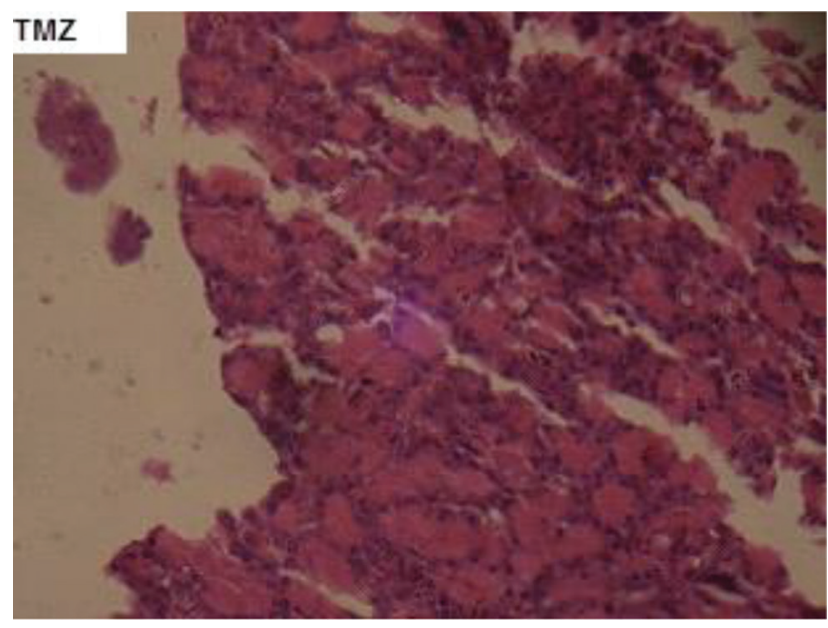

Fig. 9 Hemorrhage and fat necrosis scoring in different experimental groups.

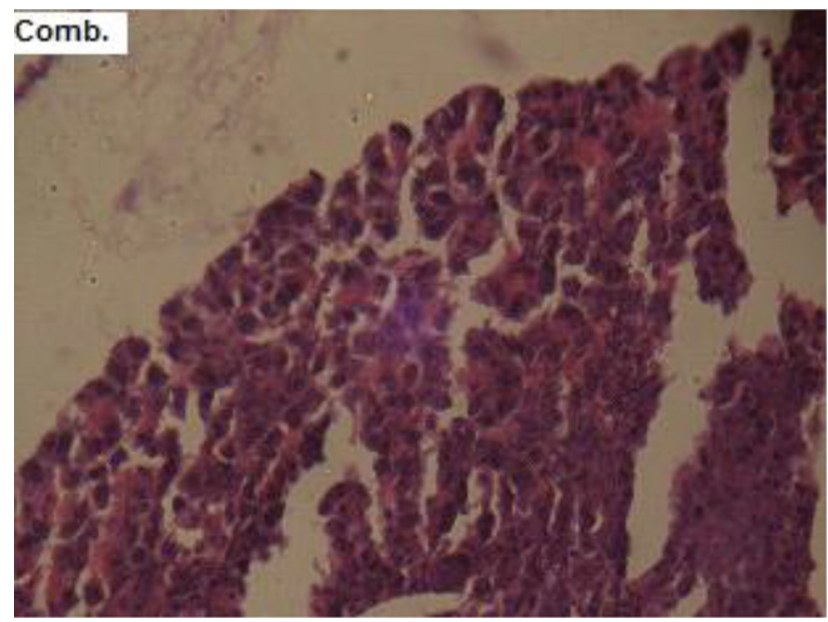

Fig. 10 Inflammation and perivascular infiltrate scoring in different experimental groups.

Also Yllmaz et al. $^{8}$ showed that there is a significant increase in IL- $1 \beta$ and TNF- $\alpha$ levels in AP induction group.

Also there is a significant decrease $(P<0.001)$ in serum levels of the TAC in induction group as compared with the control group. Similar results obtained by Cikman et al. ${ }^{9}$ regarding that there is a significant inhibition of TAC in the induction group compared to the control group and this is consistent with the hypothesis that AP may generate oxidative stress.

The oxidative stress is intensely involved in the inflammatory processes of AP since the OFRs are the major pro-oxidant agents and in the same time acts as inflammatory mediators through the stimulation of leukocytes activation, adhesion, and then emigration in addition to improving the expression of other inflammatory mediators, such as cellular adhesive molecules. ${ }^{10}$

Furthermore, Yllmaz et al. ${ }^{8}$ also shows a similar results by obtaining a low levels of TAC in the induction group compared with the control group proving the low antioxidant capacity in case of AP.

Also this study shows a significant increase $(P<0.001)$ in serum levels of the lipase and amylase in induction group as compared with the control group. Similar results were obtained by Nader and Wagih ${ }^{11}$ and Aziz et al., ${ }^{12}$ and they confirmed that there is a significant increase detected in serum levels of lipase and amylase in L-Arg treated rats compared to the control group.

Regarding the histopathological changes, induction group had significantly $(P<0.05)$ higher pancreatic tissue injury scoring as compared with the control group. Histopathological findings in induction group as well as control group were associated with edema, acinar cell necrosis, hemorrhage and fat necrosis, and inflammation and peri-vascular infiltration.

These results were similar to that obtained by Wang et al. ${ }^{7}$ showing that the pancreatic interstitial edema, leukocytes infiltrations, hemorrhage, and cellular necrosis were higher significantly in the induction group as compared with the control group.

\section{Effect of Treatment on Study Parameters after I-Arg Induction}

Effect of Treatment on Proinflammatory Cytokines (TNF-a and IL-1 $\beta$ ) after I-Arg Induction

This study shows that octreotide treatment significantly decrease the serum levels of proinflammatory cytokines (TNF- $\alpha$ and IL-1 $\beta$ ) as compared with the induction group, these findings were also obtained by Yang et al. ${ }^{13}$ who study the effects of octreotide on hepatic ischemic reperfusion injuries in a rabbit models and shows that the level of both TNF- $\alpha$ and IL- $1 \beta$ are lower significantly in octreotide group than the disease group. Also both Zhang et al. ${ }^{14}$ and Tian et al. ${ }^{15}$ proved that octreotide can significantly decrease serum TNF- $\alpha$ in acute pancreatitis.

TMZ treatment significantly decrease the serum level of proinflammatory cytokines (TNF- $\alpha$ and IL-1 $\beta$ ) as compared with the induction group, these findings were also obtained by Tanoglu et al. ${ }^{16}$ who confirmed that TMZ can significantly decrease serum TNF- $\alpha$ and IL- $1 \beta$ in the treatment of experimental sepsis in rat model.

While Tanoglu et al. ${ }^{17}$ found that serum IL- $1 \beta$ levels was significantly lower during TMZ treatment and there was no significant differences in serum TNF- $\alpha$ level, in contrary Vinokurov et al. ${ }^{18}$ show that TMZ can inhibits the secretion of TNF- $\alpha$ and may reduce the inflammatory response as a result of the blockade of secretion of proinflammatory cytokines, which may occur due to a reduced expression of the components of the receptor for endotoxin, and that is confirmed by our study that shows a significant decrease in TNF- $\alpha$ level in TMZ group as compared with both induction and octreotide group.

Combination treatment significantly decrease the serum level of proinflammatory cytokines (TNF- $\alpha$ and IL-1 $\beta$ ) as compared with the induction group.

Also there is a significant decrease in serum level of proinflammatory cytokines in combination therapy as compared with the single therapy model with either octreotide or trimetazidine alone.

According to our knowledge, there are no studies on using combination therapy with octreotide and trimetazidine in acute pancreatitis in a rat model, and this study is the first.

\section{Effect of Treatment on Total Antioxidant Capacity}

This study shows that octreotide treatment significantly increase the serum levels of TAC as compared with the induction group, these findings are similar to Wang et al..$^{19}$ that 
shows octreotide protective capacity through its ability to inhibit oxidative stress. Kara et al. ${ }^{20}$ also proves the protective effect of octreotide by significantly reducing this oxidative damage in male rats.

TMZ treatment significantly increase the serum levels of TAC as compared with the induction group, these findings were also obtained by Chen et al. ${ }^{21}$ who demonstrates that TMZ can decrease oxidative stress significantly. Similar results proved by Mahfoudh-Boussaid et al. ${ }^{22}$ regarding that TMZ significantly decrease the oxidative stress and improve renal function in Wistar rats, and also by Hassanzadeh et al. ${ }^{23}$ that concluded in their study that TMZ can avoid the oxidative stress induced structural injuries in rat hippocampus and it has a neuro-protective effect that associates with its antioxidants activity.

Our study shows a significant increase in TAC level in TMZ group as compared with octreotide group proving the strong antioxidant activity of TMZ.

Combination treatment significantly increase the serum levels of TAC as compared with the induction group.

Also there is a significant increase in serum level of TAC in combination therapy as compared with the single therapy model with either octreotide or trimetazidine alone.

According to our knowledge, there are no studies on using combination therapy with octreotide and trimetazidine in acute pancreatitis in a rat model, and this study is the first.

\section{Effect of Treatment on Pancreatic Enzymes (Lipase and Amylase)}

This study shows that octreotide treatment significantly decrease the serum level of pancreatic enzymes (amylase and lipase) as compared with the induction group, these findings were also obtained by Chen et al. ${ }^{24}$ that showed a significant decrease in lipase and amylase serum levels after octreotide treatment in experimentally induced pancreatitis in adult rats. Woeste et al. ${ }^{25}$ also showed a significant decrease in lipase level after octreotide treatment. Additionally, Kafali et al. ${ }^{26}$ proved that octreotide can significantly lower amylase level in rats in early dose immediately after AP induction. Zhou et al. ${ }^{27}$ proved that octreotide can decrease serum amylase level in canine model AP.

Our study shows a significant reduction in lipase and amylase levels in octreotide group as compared with TMZ group proving the strong anti-secretory activity of octreotide on the pancreas.

TMZ treatment significantly decrease the serum level of the pancreatic enzymes (amylase and lipase) as compared with the induction group, these findings were also obtained by Tanoglu et al. ${ }^{17}$ showing that TMZ can significantly reduce serum lipase and amylase levels in rat after induction of AP. Yenicerioglu et al. $^{5}$ also showed that TMZ significantly decreased amylase levels in L-Arg induced rats.

Combination treatment significantly decrease the serum levels of lipase and amylase as compared with the induction group.

Also there is a significant decrease in serum levels of lipase and amylase in combination therapy as compared with the single therapy model with either octreotide or trimetazidine alone.

According to our knowledge, there are no studies on using combination therapy with octreotide and trimetazidine in acute pancreatitis in a rat model, and this study is the first.

\section{Effect of Treatment on Pancreatic Tissue after I-Arg Induction}

This study shows that octreotide treatment significantly decrease the pancreatic tissue injury scoring in edema, acinar cell necrosis, hemorrhage and fat necrosis, and inflammation as compared with the induction group, a similar findings were obtained by Zhang et al. ${ }^{28,29}$ considering edema, acinar necrosis, and hemorrhage.

Also considering edema, inflammation, fat necrosis, parenchymal necrosis, and hemorrhage, Guler et al. ${ }^{30}$ found that octreotide significantly decrease the injury scoring of pancreatic tissue.

TMZ treatment significantly decrease the pancreatic tissue injury scoring in edema, acinar cell necrosis, hemorrhage and fat necrosis, and inflammation as compared with the induction group, a similar findings were obtained by Yenicerioglu et al. ${ }^{5}$ proved that TMZ significantly decrease histopathological criteria scoring of edema, acinar cell necrosis, hemorrhage and the level of perivascular inflammation in L-Arg induced AP. Additionally, Tanoglu et al. ${ }^{17}$ also found that TMZ reduce edema formation, inflammation, vacuolization and fatty necrosis of pancreatic tissues supporting the beneficial effects of TMZ treatment in experimental AP.

Combination treatment significantly decrease the serum levels of pancreatic tissue injury scoring in edema, acinar cell necrosis, hemorrhage and fat necrosis, and Inflammation as compared with the induction group.

Also there is a significant decrease in pancreatic tissue injury scoring in edema, acinar necrosis, hemorrhage and fat necrosis, and inflammation in combination therapy as compared with the single therapy model with either octreotide or TMZ alone.

According to our knowledge, there are no studies on using combination therapy with octreotide and TMZ in acute pancreatitis in a rat model, and this study is the first.

\section{Statistical Analysis}

Statistical analysis was completed by using statistical package for Social Sciences (SPSS) version 20 in which we used median, mean with standard deviation as descriptive statistics. Analysis of variance with LSD and Mann-Whitney test for comparison between groups. $P$-value $\leq 0.05$ was regarded as significant.

\section{Conclusion}

As indicated by the consequences and results of this study, we concluded that:

1. This study additionally supports the role of proinflammatory cytokine (TNF- $\alpha$ and IL- $1 \beta$ ), and oxidative stress in the pathophysiology of L-Arg induced acute pancreatitis.

2. Octreotide and trimetazidine reduce inflammatory reaction associated with $\mathrm{L}$-Arg induced acute pancreatitis as evidenced by reducing proinflammatory cytokines (TNF- $\alpha$ and IL-1 $\beta$ ).

3. Octreotide and trimetazidine shows antioxidant activity evidenced by increasing levels of the total antioxidant capacity after treatment with these drugs.

4. Octreotide has effect on pancreatic enzymes more than TMZ, while TMZ has more anti-inflammatory and 
antioxidant abilities than octreotide as shown by the results.

5. However, theeffectsofcombination therapy(octreotide plus TMZ) were superior to octreotide or TMZ alone. The combination therapy concentrated on numerous pathological pathways of AP, such as the extreme activated pancreatic enzymes, increased activity of inflammatory mechanism, and the microcirculatory dysfunction mechanism, and therefore, the effect of combination treatment is much better than monotherapy. Furthermore, it was established that TMZ can enhance the therapeutical effect of octreotide in AP in rat. Thus, the treatment of TMZ plus octreotide can be applied as a treatment of AP more comprehensively and more efficiently.

\section{Conflict of Interest}

There is no conflict of interest regarding the publication of this paper.

\section{References}

1. Bhandari M. Role of galanin and its antagonists in experimental acute pancreatitis/Mayank Bhandari, 1972. Ph.D. Thesis. Flinders University, 2008.

2. Felderbauer P, Müller C, Bulut K, Belyaev O, Schmitz F, Uhl W, et al. Pathophysiology and treatment of acute pancreatitis: new therapeutic targets-a ray of hope? Basic Clin Pharmacol Toxicol. 2005;97:342-350

3. Dawra R, Saluja AK. L-arginine-induced experimental acute pancreatitis. The Pancreapedia: The Exocrine Pancreas Knowledge Base.

4. Schmidt J, Rattner DW, Lewandrowski K, Compton CC, Mandavilli U, Knoefel WT, et al. A better model of acute pancreatitis for evaluating therapy. Ann Surg. 1992;215:44-56

5. Yenicerioglu A, Cetinkaya Z, Girgin M, Ustundag B, Ozercan IH, Ayten R, et al. Effects of trimetazidine in acute pancreatitis induced by L-arginine. Can J Surg. 2013;56:175-179.

6. Almeida JL, Sampietre SN, Mendonça Coelho AM, Trindade Molan NA, Machado MC, Monteiro da Cunha JE, et al. Statin pretreatment in experimental acute pancreatitis. JOP. 2008:9:431-439.

7. Wang Y, Guo W, Li Y, Pan X, Lv W, Cui L, et al. Hypothermia induced by adenosine 5'-monophosphate attenuates injury in an L-arginine-induced acute pancreatitis rat model. J Gastroenterol Hepatol. 2014;29:742-748.

8. Yılmaz EE, Bozdağ Z, Ibiloğlu I, Arıkanoğlu Z, Yazgan ÜC, Kaplan I, et al. Therapeutic effects of ellagic acid on L-arginin induced acute pancreatitis. Acta Cir Bras. 2016;31:396-401.

9. Cikman O, Soylemez O, Ozkan OF, Kiraz HA, Sayar I, Ademoglu S, et al. Antioxidant activity of syringic acid prevents oxidative stress in L-arginine induced acute pancreatitis: an experimental study on rats. Int Surg. 2015;100:891-896.

10. Gómez-Cambronero LG, Sabater L, Pereda J, Cassinello N, Camps B, Viña J, et al. Role of cytokines and oxidative stress in the pathophysiology of acute pancreatitis: therapeutical implications. Curr Drug Targets Inflamm Allergy. 2002;1:393-403

11. Nader MA, Wagih HM. Nilotinib, a tyrosine kinase inhibitor exhibits protection against acute pancreatitis-induced lung and liver damage in rats. Naunyn Schmiedebergs Arch Pharmacol. 2017;390:291-300.

12. Aziz NM, Kamel MY, Rifaai RA. Effects of hemin, a heme oxygenase-1 inducer in L-arginine-induced acute pancreatitis and associated lung injury in adult male albino rats. Endocr Regul. 2017:51:20-30.

13. Yang J, Sun H, Takacs P, Zhang Y, Liu J, Chang Y, et al. The effect of octreotide on hepatic ischemia-reperfusion injury in a rabbit model. Transplant Proc 2013:45:2433-2438.

14. Zhang XP, Tian H, Lai YH, Chen L, Zhang L, Cheng QH, et al. Protective effects and mechanisms of baicalin and octreotide on renal injury of rats with severe acute pancreatitis. World J Gastroenterol. 2007:13:5079-5089.

15. Tian $H$, Zhang $X$, Wu C, Chen L, Ying R, Ye J, et al. Effects of baicalin and octreotide on the serum TNF-alpha level and apoptosis in multiple organs of rats with severe acute pancreatitis. Inflammation. 2009;32:191-201.

16. Tanoglu A, Yamanel L, Inal V, Ocal R, Comert B, Bilgi C. Appreciation of trimetazidine treatment in experimental sepsis rat model. Bratisl Lek Listy. 2015;116:124-127. Trimetazidine significantly reduces cerulein-induced pancreatic apoptosis. Clin Res Hepatol Gastroenterol. 2015;39:145-150.

18. Vinokurov MG, Astashkin El, Yurinskaya MM, Glezer MG, Sobolev KE, Grachev SV. Trimetazidine blocks store-operated $\mathrm{Ca}(2+)$ channels in HL-60 and THP-1 cell lines and inhibits the secretion of tumor necrosis factor. Dokl Biol Sci. 2011:441:417-420.

19. Wang J, Sun Z, Shen J, Wu D, Liu F, Yang R, et al. Octreotide protects the mouse retina against ischemic reperfusion injury through regulation of antioxidation and activation of NF-KB. Oxid Med Cell Longev. 2015;2015:970156.

20. Kara H, Karatas F, Tug T, Canatan H, Karaoglu A. Protective effect of octreotide on intra-tracheal bleomycin-induced oxidative damage in rats. Exp Toxicol Pathol. 2010:62:235-241.

21. Chen A, Li W, Chen X, Shen Y, Dai W, Dong Q, et al. Trimetazidine attenuate pressure overload-induced early cardiac energy dysfunction via regulation of neuropeptide $Y$ system in a rat model of abdominal aortic constriction. BMC Cardiovasc Disord. 2016; 16: 225.

22. Mahfoudh-Boussaid A, Zaouali MA, Hauet T, Hadj-Ayed K, Miled AH, Ghoul-Mazgar S, et al. Attenuation of endoplasmic reticulum stress and mitochondrial injury in kidney with ischemic postconditioning application and trimetazidine treatment. J Biomed Sci. 2012;19:71.

23. Hassanzadeh G, Hosseini A, Pasbakhsh P, Akbari M, Ghaffarpour M, Takzare N, et al. Trimetazidine prevents oxidative changes induced in a rat model of sporadic type of Alzheimer's disease. Acta Med Iran. 2015;53:17-24

24. Chen CC, Wang SS, Lee FY, Tsay SH, Wu SL, Lu RH, et al. Prophylactic octreotide reduces the severity of histopathologic changes and hemodynamic shock in early taurodeoxycholate-induced experimental pancreatitis. Proc Natl Sci Counc Repub China B. 1999;23:1-6.

25. Woeste G, Wullstein C, Meyer S, Usadel KH, Hopt UT, Bechstein WO, et al. Octreotide attenuates impaired microcirculation in postischemic pancreatitis when administered before induction of ischemia. Transplantation. 2008:86:961-967.

26. Kafali ME, Gul M, Alptekin H, Sahin M, Toy H, Akoz M. Is there a relationship between beginning time and efficiency of octreotide in the treatment of experimental acute pancreatitis? J Korean Surg Soc. 2012;82:296-301.

27. Zhou MT, Chen BC, Sun HW, Jin YP, Yang FJ, Zhang X, et al. Continuous regional arterial infusion with fluorouracil and octreotide attenuates severe acute pancreatitis in a canine model. PLoS One. 2012;7:e37347.

28. Zhang XP, Zhang L, Yang P, Zhang RP, Cheng QH. Protective effects of baicalin and octreotide on multiple organ injury in severe acute pancreatitis. Dig Dis Sci. 2008:53:581-591.

29. Zhang XP, Tian H, Wu DJ, Feng GH, Chen L, Zhang J, et al. Pathological changes in multiple organs of rats with severe acute pancreatitis treated by baicalin and octreotide. Hepatobiliary Pancreat Dis Int. 2009;8:85-92.

30. Güler O, Akturan S, Kisli E, Dolapçi I, Caydere M, Akova A. Acute pancreatitis, bacterial translocation, and different octreotide regimens: an experimental study. Surg Today. 2009;39:876-883 